\title{
Solvability of a Quadratic Integral Equation of Fredholm Type with Supremum in Hölder Spaces
}

\author{
J. Caballero Mena, ${ }^{1}$ R. Nalepa, ${ }^{2}$ and K. Sadarangani ${ }^{1}$ \\ ${ }^{1}$ Department of Mathematics, University of Las Palmas de Gran Canaria, Campus de Tafira Baja, \\ 35017 Las Palmas de Gran Canaria, Spain \\ ${ }^{2}$ Department of Mathematics, Rzeszów University of Technology, Aleja Powstańców Warszawy 12, 35-959 Rzeszów, Poland
}

Correspondence should be addressed to R. Nalepa; r_nalepa@prz.edu.pl

Received 14 December 2013; Accepted 27 January 2014; Published 29 April 2014

Academic Editor: Józef Banaś

Copyright (c) 2014 J. Caballero Mena et al. This is an open access article distributed under the Creative Commons Attribution License, which permits unrestricted use, distribution, and reproduction in any medium, provided the original work is properly cited.

Using the classical Schauder fixed point theorem, we prove the existence of solutions of a quadratic integral equation of Fredholm type with supremum in the space of functions satisfying the Hölder condition.

\section{Introduction}

Quadratic integral equations arise naturally in applications of real-world problems. For example, problems in the theory of radiative transfer in the theory of neutron transport and in the kinetic theory of gases lead to the quadratic equation

$$
x(t)=1+t x(t) \int_{0}^{1} \frac{\Phi(s)}{t+s} x(s) d s,
$$

where $\Phi$ is a continuous function defined on the interval $[0,1]$ (see [1-4]).

Equations of this type have been studied by several authors [5-11].

The aim of this paper is to investigate the existence of solutions of the following quadratic integral equation of Fredholm type with supremum:

$$
\begin{aligned}
& x(t) \\
& =p(t)+x(t) \int_{0}^{1} k(t, \tau)\left\{\max _{\eta \in[0, r(\tau)]}|x(\eta)|\right\} d \tau, \quad t \in[0,1] .
\end{aligned}
$$

Differential and integral equations with supremum are adequate models of real-world problems, in which the present state depends significantly on this maximum value on a past time interval [12]. Equations of such kind have been studied in some papers appearing in the literature (see [13-20]).

Our solutions are placed in the space of functions satisfying the Hölder condition. A sufficient condition for the relative compactness in these spaces and the classical Schauder fixed point theorem are the main tools used in our study.

\section{Preliminaries}

Our starting point in this section is to introduce the space of functions satisfying the Hölder condition and some properties in this space. These properties appear in [21].

Let $[a, b]$ be a closed interval in $\mathbb{R}$; by $C[a, b]$ we denote the space of the continuous functions on $[a, b]$ equipped with the norm $\|x\|_{\infty}=\sup \{|x(t)|: t \in[a, b]\}$.

For fixed $0<\alpha \leqslant 1$, by $H_{\alpha}[a, b]$, we will denote the space of the real functions $x$ defined on $[a, b]$ and satisfying the Hölder condition, that is, those functions $x$ for which there exists a constant $H_{x}^{\alpha}$ such that

$$
|x(t)-x(s)| \leqslant H_{x}^{\alpha}|t-s|^{\alpha},
$$

for all $t, s \in[a, b]$.

It is easily seen that $H_{\alpha}[a, b]$ forms a linear subspace of $C[a, b]$. 
In what follows, for $x \in H_{\alpha}[a, b]$, by $H_{x}^{\alpha}$, we will denote the least possible constant for which inequality (3) is satisfied. More "precisely," we put

$$
H_{x}^{\alpha}=\sup \left\{\frac{|x(t)-x(s)|}{|t-s|^{\alpha}}: t, s \in[a, b], t \neq s\right\} .
$$

The space $H_{\alpha}[a, b]$ with $0<\alpha \leqslant 1$ can be normed under the following norm:

$$
\begin{aligned}
\|x\|_{\alpha}= & |x(a)| \\
& +\sup \left\{\frac{|x(t)-x(s)|}{|t-s|^{\alpha}}: t, s \in[a, b], t \neq s\right\},
\end{aligned}
$$

for $x \in H_{\alpha}[a, b]$.

It is proved in [21] that $\left(H_{\alpha}[a, b],\|\cdot\|_{\alpha}\right)$ with $0<\alpha \leqslant 1$ is a Banach space.

Now, we recollect some results about the spaces $H_{\alpha}[a, b]$ with $0<\alpha \leqslant 1$ which appear in [21].

Lemma 1. For $x \in H_{\alpha}[a, b]$ with $0<\alpha \leqslant 1$ the following inequality is satisfied:

$$
\|x\|_{\infty} \leqslant \max \left(1,(b-a)^{\alpha}\right)\|x\|_{\alpha} .
$$

Lemma 2. For $0<\alpha<\gamma \leqslant 1$, one has

$$
H_{\gamma}[a, b] \subset H_{\alpha}[a, b] \subset C[a, b] .
$$

Moreover, for $x \in H_{\gamma}[a, b]$ the following inequality holds:

$$
\|x\|_{\alpha} \leqslant \max \left(1,(b-a)^{\gamma-\alpha}\right)\|x\|_{\gamma}
$$

The following sufficient condition for relative compactness in the spaces $H_{\alpha}[a, b]$ with $0<\alpha \leqslant 1$ appears in Example 6 of [21].

Theorem 3. Suppose that $0<\alpha<\beta \leqslant 1$ and that $A$ is a bounded subset in $H_{\beta}[a, b]$ (this means that there exists a constant $M>0$ such that $|x(t)-x(s)| \leqslant M|t-s|^{\beta}$ for any $x \in A$ and for any $t, s \in[a, b])$. Then $A$ is a relatively compact subset of $H_{\alpha}[a, b]$.

Remark 4. Suppose that $0<\alpha<\beta \leqslant 1$ and by $B_{r}^{\beta}$ we denote the ball centered at $\theta$ and radius $r$ in the space $H_{\beta}[a, b]$; that is, $B_{r}^{\beta}=\left\{x \in H_{\beta}[a, b]:\|x\|_{\beta} \leqslant r\right\}$. Then $B_{r}^{\beta}$ is compact in the space $H_{\alpha}[a, b]$.

Proof. In fact, by Theorem 3 , since $B_{r}^{\beta}$ is a bounded subset in $H_{\beta}[a, b], B_{r}^{\beta}$ is a relatively compact subset of $H_{\alpha}[a, b]$.

Suppose that $\left(x_{n}\right) \subset B_{r}^{\beta}$ and $x_{n} \stackrel{\|\cdot\|_{\alpha}}{\longrightarrow} x$ with $x \in H_{\alpha}[a, b]$. This means that for $\varepsilon>0$ we can find $n_{0} \in \mathbb{N}$ such that

$$
\left\|x_{n}-x\right\|_{\alpha} \leqslant \varepsilon \quad \text { for any } n \geqslant n_{0}
$$

or, equivalently

$$
\begin{gathered}
\left|x_{n}(a)-x(a)\right| \\
+\sup \left\{\frac{\left|x_{n}(t)-x(t)-\left(x_{n}(s)-x(s)\right)\right|}{|t-s|^{\alpha}}:\right. \\
\quad t, s \in[a, b], t \neq s\} \leqslant \varepsilon
\end{gathered}
$$

for any $n \geqslant n_{0}$.

This implies that $x_{n}(a) \rightarrow x(a)$.

Moreover, if in (10) we put $s=a$, then we get

$\sup \left\{\frac{\left|x_{n}(t)-x(t)-\left(x_{n}(a)-x(a)\right)\right|}{|t-a|^{\alpha}}: t \in[a, b], t \neq a\right\}<\varepsilon$ for any $n \geqslant n_{0}$.

The last inequality implies that

$$
\begin{aligned}
& \left|x_{n}(t)-x(t)-\left(x_{n}(a)-x(a)\right)\right|<\varepsilon|t-a|^{\alpha} \leqslant \varepsilon(b-a)^{\alpha} \\
& \text { for any } n \geqslant n_{0} \text { and for any } t \in[a, b] .
\end{aligned}
$$

Therefore, for any $n \geqslant n_{0}$ and any $t \in[a, b]$ and taking into account (10) and (12), we have

$$
\begin{aligned}
\left|x_{n}(t)-x(t)\right| \leqslant & \left|\left(x_{n}(t)-x(t)\right)-\left(x_{n}(a)-x(a)\right)\right| \\
& +\left|x_{n}(a)-x(a)\right|<\varepsilon(b-a)^{\alpha}+\varepsilon \\
= & \varepsilon\left(1+(b-a)^{\alpha}\right) .
\end{aligned}
$$

Consequently,

$$
\left\|x_{n}-x\right\|_{\infty} \longrightarrow 0
$$

Next, we will prove that $x \in B_{r}^{\beta}$.

In fact, since $\left(x_{n}\right) \subset B_{r}^{\beta} \subset H_{\beta}[a, b]$, we have that

$$
\frac{\left|x_{n}(t)-x_{n}(s)\right|}{|t-s|^{\beta}} \leqslant r \quad \text { for any } t, s \in[a, b] \text { with } t \neq s,
$$

and, accordingly,

$$
\left|x_{n}(t)-x_{n}(s)\right| \leqslant r|t-s|^{\beta} \quad \text { for any } t, s \in[a, b] .
$$

Letting $n \rightarrow \infty$ in the above inequality and taking into account (14), we deduce that

$$
|x(t)-x(s)| \leqslant r|t-s|^{\beta} \quad \text { for any } t, s \in[a, b] \text {. }
$$

Hence, we get

$$
\frac{|x(t)-x(s)|}{|t-s|^{\beta}} \leqslant r \quad \text { for any } t, s \in[a, b],
$$

and this means that $x \in B_{r}^{\beta}$.

This proves that $B_{r}^{\beta}$ is a closed subset of $H_{\alpha}[a, b]$. Thus, $B_{r}^{\beta}$ is a compact subset of $H_{\alpha}[a, b]$.

This finishes the proof.

Next, we recall some results appearing in [18].

In what follows, we consider $[a, b]=[0,1]$. 
Lemma 5. Let $r:[0,1] \rightarrow[0,1]$ be a continuous and nondecreasing function and $x \in C[0,1]$. Let $G x$ be the function defined by

$$
(G x)(t)=\max _{\tau \in[0, r(t)]}|x(\tau)| \quad \text { for } t \in[0,1] .
$$

Then $G x \in C[0,1]$.

Under the above assumption we have the following.

Lemma 6. Let $\left(x_{n}\right)$ be a sequence in $C[0,1]$ and $x \in C[0,1]$ such that $x_{n} \rightarrow x$ in $C[0,1]$. Then

$$
\left\|G x_{n}-G x\right\|_{\infty} \leqslant\left\|x_{n}-x\right\|_{\infty} .
$$

Finally, we recall Schauder's fixed point theorem.

Theorem 7. Let $\mathrm{Q}$ be a nonempty, convex, and compact subset of a Banach space $(X,\|\cdot\|)$ and let $T: Q \rightarrow Q$ be a continuity mapping. Then $T$ has at least one fixed point in $Q$.

\section{Main Result}

In this section we will investigate the solvability of the integral equation (2) in the Hölder spaces.

We will formulate the following assumptions:

(i) $p \in H_{\beta}[0,1](0<\beta \leqslant 1)$;

(ii) $k:[0,1] \times[0,1] \rightarrow \mathbb{R}$ is a continuous function such that it satisfies the Hölder condition with exponent $\beta$ with respect to the first variable; that is, there exists a constant $k_{\beta}>0$ such that

$$
|k(t, \varrho)-k(s, \varrho)| \leqslant k_{\beta}|t-s|^{\beta},
$$

for any $t, s, \varrho \in[0,1]$;

(iii) $r:[0,1] \rightarrow[0,1]$ is a continuous and nondecreasing function;

(iv) the following inequality is satisfied:

$$
\|p\|_{\beta}\left(2 K+k_{\beta}\right)<\frac{1}{4},
$$

where the constant $K$ is defined by

$$
K=\sup \left\{\int_{0}^{1}|k(t, \tau)| d \tau: t \in[0,1]\right\}
$$

and whose existence is guaranteed by virtue of (ii).

Theorem 8. Under assumptions (i)-(iv), (2) has at least one solution belonging to the space $H_{\alpha}[0,1]$, where $\alpha$ is arbitrarily fixed number satisfying $0<\alpha<\beta$.

Proof. Let us consider the operator $F$ defined on the space $H_{\beta}[0,1]$ by

$$
(F x)(t)=p(t)+x(t) \int_{0}^{1} k(t, \tau)\left\{\max _{\eta \in[0, r(\tau)]}|x(\eta)|\right\} d \tau,
$$

for $t \in[0,1]$.
First, we will prove that $F$ transforms the space $H_{\beta}[0,1]$ into itself.

In fact, we take an arbitrary function $x \in H_{\beta}[0,1]$ and $t, s \in[0,1]$ such that $t \neq s$. Then, in view of assumptions (i) and (ii), we get

$$
\begin{aligned}
& \frac{|(F x)(t)-(F x)(s)|}{|t-s|^{\beta}} \\
& =\mid p(t)+x(t) \int_{0}^{1} k(t, \tau)\left\{\max _{\eta \in[0, r(\tau)]}|x(\eta)|\right\} d \tau \\
& -p(s)-x(s) \int_{0}^{1} k(s, \tau)\left\{\max _{\eta \in[0, r(\tau)]}|x(\eta)|\right\} d \tau \mid \\
& \times\left(|t-s|^{\beta}\right)^{-1} \\
& \leqslant \frac{|p(t)-p(s)|}{|t-s|^{\beta}} \\
& +\mid x(t) \int_{0}^{1} k(t, \tau)\left\{\max _{\eta \in[0, r(\tau)]}|x(\eta)|\right\} d \tau-x(s) \\
& \times \int_{0}^{1} k(t, \tau)\left\{\max _{\eta \in[0, r(\tau)]}|x(\eta)|\right\} d \tau \mid \\
& \times\left(|t-s|^{\beta}\right)^{-1} \\
& +\mid x(s) \int_{0}^{1} k(t, \tau)\left\{\max _{\eta \in[0, r(\tau)]}|x(\eta)|\right\} d \tau \\
& -x(s) \int_{0}^{1} k(s, \tau)\left\{\max _{\eta \in[0, r(\tau)]}|x(\eta)|\right\} d \tau \mid \\
& \times\left(|t-s|^{\beta}\right)^{-1} \\
& \leqslant \frac{|p(t)-p(s)|}{|t-s|^{\beta}}+\frac{|x(t)-x(s)|}{|t-s|^{\beta}} \\
& \cdot \int_{0}^{1}|k(t, \tau)|\left\{\max _{\eta \in[0, r(\tau)]}|x(\eta)|\right\} d \tau \\
& +\frac{|x(s)| \int_{0}^{1}|k(t, \tau)-k(s, \tau)|\left\{\max _{\eta \in[0, r(\tau)]}|x(\eta)|\right\} d \tau}{|t-s|^{\beta}} \\
& \leqslant \frac{|p(t)-p(s)|}{|t-s|^{\beta}}+\frac{|x(t)-x(s)|}{|t-s|^{\beta}}\|x\|_{\infty} \\
& \times \int_{0}^{1}|k(t, \tau)| d \tau \\
& +\frac{\|x\|_{\infty}\|x\|_{\infty} \int_{0}^{1}|k(t, \tau)-k(s, \tau)| d \tau}{|t-s|^{\beta}} \\
& \leqslant \frac{|p(t)-p(s)|}{|t-s|^{\beta}}+K\|x\|_{\infty}
\end{aligned}
$$




$$
\begin{aligned}
& \times \frac{|x(t)-x(s)|}{|t-s|^{\beta}}+\frac{\|x\|_{\infty}^{2} \int_{0}^{1} k_{\beta}|t-s|^{\beta} d \tau}{|t-s|^{\beta}} \\
\leqslant & H_{p}^{\beta}+K\|x\|_{\infty} H_{x}^{\beta}+k_{\beta}\|x\|_{\infty}^{2} .
\end{aligned}
$$

By Lemma $1,\|x\|_{\infty} \leqslant\|x\|_{\beta}$, and, since $H_{x}^{\beta} \leqslant\|x\|_{\beta}$, from the last inequality it follows that

$$
\begin{aligned}
\frac{|(F x)(t)-(F x)(s)|}{|t-s|^{\beta}} & \leqslant H_{p}^{\beta}+K\|x\|_{\beta}^{2}+k_{\beta}\|x\|_{\beta}^{2} \\
& =H_{p}^{\beta}+\left(K+k_{\beta}\right)\|x\|_{\beta}^{2} .
\end{aligned}
$$

Therefore,

$$
\begin{aligned}
\|F x\|_{\beta} & |(F x)(0)|+\sup \left\{\frac{|(F x)(t)-(F x)(s)|}{|t-s|^{\beta}}: t, s \in[0,1], t \neq s\right\} \\
\leqslant & |(F x)(0)|+H_{p}^{\beta}+\left(K+k_{\beta}\right)\|x\|_{\beta}^{2} \\
\leqslant & |p(0)|+|x(0)| \int_{0}^{1}|k(0, \tau)|\left\{\max _{\eta \in[0, r(\tau)]}|x(\eta)|\right\} d \tau \\
& +H_{p}^{\beta}+\left(K+k_{\beta}\right)\|x\|_{\beta}^{2} \\
\leqslant & \|p\|_{\beta}+\|x\|_{\infty} \cdot\|x\|_{\infty} \int_{0}^{1}|k(0, \tau)| d \tau \\
& +\left(K+k_{\beta}\right)\|x\|_{\beta}^{2} \leqslant\|p\|_{\beta}+K\|x\|_{\infty}^{2}+\left(K+k_{\beta}\right)\|x\|_{\beta}^{2} \\
\leqslant & \|p\|_{\beta}+K\|x\|_{\beta}^{2}+\left(K+k_{\beta}\right)\|x\|_{\beta}^{2} \\
= & \|p\|_{\beta}+\left(2 K+k_{\beta}\right)\|x\|_{\beta}^{2}<\infty,
\end{aligned}
$$

where we have used the fact that $\|p\|_{\beta}=|p(0)|+H_{p}^{\beta}$.

This shows that the operator $F$ transforms $H_{\beta}[0,1]$ into itself.

On the other hand, the inequality

$$
\|p\|_{\beta}+\left(2 K+k_{\beta}\right) r^{2} \leqslant r
$$

is satisfied by the number

$$
\begin{aligned}
& r_{1}=\frac{1-\sqrt{1-4\|p\|_{\beta}\left(2 K+k_{\beta}\right)}}{2\left(2 K+k_{\beta}\right)}, \\
& r_{2}=\frac{1+\sqrt{1-4\|p\|_{\beta}\left(2 K+k_{\beta}\right)}}{2\left(2 K+k_{\beta}\right)},
\end{aligned}
$$

which are positive by virtue of (iv), and, consequently, from (27) we infer that $F$ transforms the ball $B_{r_{0}}^{\beta}=\left\{x \in H_{\beta}[0,1]\right.$ : $\left.\|x\|_{\beta} \leqslant r_{0}\right\}$ into itself, for any $r_{0} \in\left[r_{1}, r_{2}\right]$.
Therefore, $F: B_{r_{0}}^{\beta} \rightarrow B_{r_{0}}^{\beta}$, where $r_{1} \leqslant r_{0} \leqslant r_{2}$.

By Theorem 3 and Remark $4, B_{r_{0}}^{\beta}$ is a compact subset in the space $H_{\alpha}[0,1]$ for any $0<\alpha<\beta \leqslant 1$.

Next, we will prove that the operator $F$ is continuous on $B_{r_{0}}^{\beta}$, where in $B_{r_{0}}^{\beta}$ we consider the induced norm by $\|\cdot\|_{\alpha}$, where $0<\alpha<\beta \leqslant 1$.

To this end fix $x \in B_{r_{0}}^{\beta}$ and $\varepsilon>0$. Suppose that $y \in B_{r_{0}}^{\beta}$ and $\|x-y\|_{\alpha} \leqslant \delta$, where $\delta \leqslant \varepsilon / 2\left(2 K+3 k_{\beta}\right) r_{0}$.

Then, for any $t, s \in[0,1]$ with $t \neq s$, we have

$\frac{|[(F x)(t)-(F y)(t)]-[(F x)(s)-(F y)(s)]|}{|t-s|^{\alpha}}$

$=\mid\left[x(t) \int_{0}^{1} k(t, \tau)\left\{\max _{\eta \in[0, r(\tau)]}|x(\eta)|\right\} d \tau\right.$

$\left.-y(t) \int_{0}^{1} k(t, \tau)\left\{\max _{\eta \in[0, r(\tau)]}|y(\eta)|\right\} d \tau\right]$

$-\left[x(s) \int_{0}^{1} k(s, \tau)\left\{\max _{\eta \in[0, r(\tau)]}|x(\eta)|\right\} d \tau\right.$

$\left.-y(s) \int_{0}^{1} k(s, \tau)\left\{\max _{\eta \in[0, r(\tau)]}|y(\eta)|\right\} d \tau\right] \mid$

$\times\left(|t-s|^{\alpha}\right)^{-1}$

$=\mid\left[x(t) \int_{0}^{1} k(t, \tau)\left\{\max _{\eta \in[0, r(\tau)]}|x(\eta)|\right\} d \tau\right.$

$\left.-y(t) \int_{0}^{1} k(t, \tau)\left\{\max _{\eta \in[0, r(\tau)]}|x(\eta)|\right\} d \tau\right]$

$+\left[y(t) \int_{0}^{1} k(t, \tau)\left\{\max _{\eta \in[0, r(\tau)]}|x(\eta)|\right\} d \tau\right.$

$$
\left.-y(t) \int_{0}^{1} k(t, \tau)\left\{\max _{\eta \in[0, r(\tau)]}|y(\eta)|\right\} d \tau\right]
$$

$-\left[x(s) \int_{0}^{1} k(s, \tau)\left\{\max _{\eta \in[0, r(\tau)]}|x(\eta)|\right\} d \tau\right.$

$\left.-y(s) \int_{0}^{1} k(s, \tau)\left\{\max _{\eta \in[0, r(\tau)]}|x(\eta)|\right\} d \tau\right]$

$-\left[y(s) \int_{0}^{1} k(s, \tau)\left\{\max _{\eta \in[0, r(\tau)]}|x(\eta)|\right\} d \tau\right.$

$\left.-y(s) \int_{0}^{1} k(s, \tau)\left\{\max _{\eta \in[0, r(\tau)]}|y(\eta)|\right\} d \tau\right] \mid$

$\times\left(|t-s|^{\alpha}\right)^{-1}$

$=\mid[x(t)-y(t)] \int_{0}^{1} k(t, \tau)\left\{\max _{\eta \in[0, r(\tau)]}|x(\eta)|\right\} d \tau$

$+y(t) \int_{0}^{1} k(t, \tau)\left\{\max _{\eta \in[0, r(\tau)]}|x(\eta)|-\max _{\eta \in[0, r(\tau)]}|y(\eta)|\right\} d \tau$ 


$$
\begin{aligned}
& -[x(s)-y(s)] \int_{0}^{1} k(s, \tau)\left\{\max _{\eta \in[0, r(\tau)]}|x(\eta)|\right\} d \tau \\
& -y(s) \int_{0}^{1} k(s, \tau)\left\{\max _{\eta \in[0, r(\tau)]}|x(\eta)|-\max _{\eta \in[0, r(\tau)]}|y(\eta)|\right\} d \tau \mid \\
& \frac{1}{|t-s|^{\alpha}} \\
& \leqslant\{|[x(t)-y(t)]-[x(s)-y(s)]| \\
& \cdot\left|\int_{0}^{1} k(t, \tau)\left\{\max _{\eta \in[0, r(\tau)]}|x(\eta)|\right\} d \tau\right|+|x(s)-y(s)| \\
& \cdot\left|\int_{0}^{1}(k(t, \tau)-k(s, \tau))\left\{\max _{\eta \in[0, r(\tau)]}|x(\eta)|\right\} d \tau\right| \\
& +\mid y(t) \int_{0}^{1} k(t, \tau)\left\{\max _{\eta \in[0, r(\tau)]}|x(\eta)|\right. \\
& \left.-\max _{\eta \in[0, r(\tau)]}|y(\eta)|\right\} d \tau-y(s) \\
& \left.\times \int_{0}^{1} k(s, \tau)\left\{\max _{\eta \in[0, r(\tau)]}|x(\eta)|-\max _{\eta \in[0, r(\tau)]}|y(\eta)|\right\} d \tau \mid\right\} \\
& \frac{1}{|t-s|^{\alpha}} \\
& \leqslant \frac{|[x(t)-y(t)]-[x(s)-y(s)]|}{|t-s|^{\alpha}} \\
& \cdot\|x\|_{\infty} \int_{0}^{1}|k(t, \tau)| d \tau \\
& +[|[x(s)-y(s)]-[x(0)-y(0)]| \\
& +|x(0)-y(0)|] \cdot\|x\|_{\infty} \\
& \times \int_{0}^{1} \frac{|k(t, \tau)-k(s, \tau)|}{|t-s|^{\alpha}} d \tau \\
& +\mid y(t) \int_{0}^{1} k(t, \tau)\left\{\max _{\eta \in[0, r(\tau)]}|x(\eta)|-\max _{\eta \in[0, r(\tau)]}|y(\eta)|\right\} d \tau \\
& -y(s) \int_{0}^{1} k(t, \tau)\left\{\max _{\eta \in[0, r(\tau)]}|x(\eta)|-\max _{\eta \in[0, r(\tau)]}|y(\eta)|\right\} d \tau \mid \\
& \frac{1}{|t-s|^{\alpha}} \\
& +\mid y(s) \int_{0}^{1} k(t, \tau)\left\{\max _{\eta \in[0, r(\tau)]}|x(\eta)|-\max _{\eta \in[0, r(\tau)]}|y(\eta)|\right\} d \tau \\
& -y(s) \int_{0}^{1} k(s, \tau)\left\{\max _{\eta \in[0, r(\tau)]}|x(\eta)|-\max _{\eta \in[0, r(\tau)]}|y(\eta)|\right\} d \tau \mid \\
& \frac{1}{|t-s|^{\alpha}}
\end{aligned}
$$$$
\begin{aligned}
\leqslant & \|x-y\|_{\alpha}\|x\|_{\infty} K \\
& +\sup _{t, s \in[0,1]}|[x(t)-y(t)]-[x(s)-y(s)]| \\
& \cdot\|x\|_{\infty} \cdot \int_{0}^{1} \frac{k_{\beta}|t-s|^{\beta}}{|t-s|^{\alpha}} d \tau+|x(0)-y(0)| \cdot\|x\|_{\infty} \\
& \times \int_{0}^{1} \frac{k_{\beta}|t-s|^{\beta}}{|t-s|^{\alpha}} d \tau+\frac{|y(t)-y(s)|}{|t-s|^{\alpha}} \\
& \times \int_{0}^{1}|k(t, \tau)|\left|\left\{\max _{\eta \in[0, r(\tau)]}|x(\eta)|-\max _{\eta \in[0, r(\tau)]}|y(\eta)|\right\}\right| d \tau \\
& +|y(s)| \int_{0}^{1} \frac{|k(t, \tau)-k(s, \tau)|}{|t-s|^{\alpha}} \\
& \times\left|\left\{\max _{\eta \in[0, r(\tau)]}|x(\eta)|-\max _{\eta \in[0, r(\tau)]}|y(\eta)|\right\}\right| d \tau
\end{aligned}
$$$$
\leqslant K\|x\|_{\infty}\|x-y\|_{\alpha}
$$$$
+\sup _{t, s \in[0,1]}\left\{\frac{|[x(t)-y(t)]-[x(s)-y(s)]|}{|t-s|^{\alpha}} \cdot|t-s|^{\alpha}\right\}
$$$$
\cdot\|x\|_{\infty} k_{\beta}|t-s|^{\beta-\alpha}+k_{\beta}\|x\|_{\infty}|t-s|^{\beta-\alpha}|x(0)-y(0)|
$$$$
+H_{y}^{\alpha} K\|x-y\|_{\infty}+\|y\|_{\infty}\|x-y\|_{\infty} \int_{0}^{1} \frac{k_{\beta}|t-s|^{\beta}}{|t-s|^{\alpha}} d \tau
$$$$
\leqslant K\|x\|_{\beta}\|x-y\|_{\alpha}+k_{\beta}\|x\|_{\beta}\|x-y\|_{\alpha}
$$$$
+k_{\beta}\|x\|_{\beta}\|x-y\|_{\alpha}+H_{y}^{\alpha} K\|x-y\|_{\alpha}
$$$$
+k_{\beta}\|y\|_{\beta}\|x-y\|_{\alpha}
$$$$
\leqslant\left(K r_{0}+3 k_{\beta} r_{0}\right)\|x-y\|_{\alpha}
$$$$
+K\|y\|_{\alpha}\|x-y\|_{\alpha} \leqslant\left(K r_{0}+3 k_{\beta} r_{0}\right)
$$$$
\times\|x-y\|_{\alpha}+K\|y\|_{\beta}\|x-y\|_{\alpha}
$$$$
\leqslant\left(2 K r_{0}+3 k_{\beta} r_{0}\right)\|x-y\|_{\alpha}
$$$$
\leqslant\left(2 K r_{0}+3 k_{\beta} r_{0}\right) \delta<\frac{\varepsilon}{2} \text {. }
$$

On the other hand, we have

$$
\begin{aligned}
& |(F x)(0)-(F y)(0)| \\
& =\mid x(0) \int_{0}^{1} k(0, \tau)\left\{\max _{\eta \in[0, r(\tau)]}|x(\eta)|\right\} d \tau \\
& \quad-y(0) \int_{0}^{1} k(0, \tau)\left\{\max _{\eta \in[0, r(\tau)]}|y(\eta)|\right\} d \tau \mid
\end{aligned}
$$




$$
\begin{aligned}
\leqslant & \mid x(0) \int_{0}^{1} k(0, \tau)\left\{\max _{\eta \in[0, r(\tau)]}|x(\eta)|\right\} d \tau \\
& -x(0) \int_{0}^{1} k(0, \tau)\left\{\max _{\eta \in[0, r(\tau)]}|y(\eta)|\right\} d \tau \mid \\
& +\mid x(0) \int_{0}^{1} k(0, \tau)\left\{\max _{\eta \in[0, r(\tau)]}|y(\eta)|\right\} d \tau \\
& \quad-y(0) \int_{0}^{1} k(0, \tau)\left\{\max _{\eta \in[0, r(\tau)]}|y(\eta)|\right\} d \tau \mid \\
\leqslant & \left|x(0) \int_{0}^{1} k(0, \tau)\left\{\max _{\eta \in[0, r(\tau)]}|x(\eta)|-\max _{\eta \in[0, r(\tau)]}|y(\eta)|\right\} d \tau\right| \\
& +\left|[x(0)-y(0)] \int_{0}^{1} k(0, \tau)\left\{\max _{\eta \in[0, r(\tau)]}|y(\eta)|\right\} d \tau\right| \\
\leqslant & \|x\|_{\infty} K\|x-y\|_{\infty}+\|x-y\|_{\alpha}\|y\|_{\infty} K \\
\leqslant & \|x\|_{\beta} K\|x-y\|_{\alpha}+\|x-y\|_{\alpha}\|y\|_{\beta} K \\
\leqslant & 2 K r_{0}\|x-y\|_{\alpha}<2 K r_{0} \delta<\frac{\varepsilon}{2} .
\end{aligned}
$$

From (30) and (31), we infer that

$$
\begin{aligned}
& \|F x-F y\|_{\alpha} \\
& =|(F x)(0)-(F y)(0)| \\
& +\sup \left\{\frac{|[(F x)(t)-(F y)(t)]-[(F x)(s)-(F y)(s)]|}{|t-s|^{\alpha}}:\right. \\
& t, s \in[0,1], t \neq s\} \\
& <\frac{\varepsilon}{2}+\frac{\varepsilon}{2}=\varepsilon .
\end{aligned}
$$

This shows that the operator $F$ is continuous at the point $x \in$ $B_{r_{0}}^{\beta}$, where $x$ is arbitrary. result.

Finally, applying Theorem 7 , we deduce that desired

Finally, we illustrate our results by presenting an example.

Example 9. Let us consider the following quadratic integral equation:

$$
\begin{aligned}
x(t)= & \sqrt{q t+r}+x(t) \\
& \times \int_{0}^{1} \sqrt[3]{m t^{2}+\tau}\left\{\max _{\eta \in[0, \tau /(\tau+1)]}|x(\eta)|\right\} d \tau,
\end{aligned}
$$

where $t \in[0,1]$ and $q, r$, and $m$ are positive constants.

Notice that (33) is a particular case of $(2)$, where $p(t)=$ $\sqrt{q t+r}, k(t, \tau)=\sqrt[3]{m t^{2}+\tau}$ and $r(\tau)=\tau /(\tau+1)$.
It is easily seen that

$$
|p(t)-p(s)| \leqslant \sqrt{q}|t-s|^{1 / 2}, \quad \text { for any } t, s \in[0,1] \text {, }
$$

and, consequently, $p(t)=\sqrt{q t+r} \in H_{1 / 2}[0,1]$.

Moreover, using the inequality proved in [11], we have

$$
\begin{aligned}
|k(t, \tau)-k(s, \tau)| & =\left|\sqrt[3]{m t^{2}+\tau}-\sqrt[3]{m s^{2}+\tau}\right| \\
& \leqslant \sqrt[3]{m}|t-s|^{2 / 3}
\end{aligned}
$$

for any $t, s, \tau \in[0,1]$. Therefore, assumption (ii) of Theorem 8 is satisfied, since for any $t, s, \tau \in[0,1]$

$$
\begin{aligned}
& |k(t, \tau)-k(s, \tau)| \leqslant \sqrt[3]{m}|t-s|^{2 / 3} \\
& \quad=\sqrt[3]{m}|t-s|^{1 / 2}|t-s|^{1 / 6} \leqslant \sqrt[3]{m}|t-s|^{1 / 2}
\end{aligned}
$$

where $k_{\beta}=\sqrt[3]{m}$.

Moreover, we have that

$$
\begin{aligned}
\|p\|_{1 / 2} & =|p(0)|+\sup \left\{\frac{|p(t)-p(s)|}{|t-s|^{1 / 2}}: t, s \in[0,1], t \neq s\right\} \\
& =\sqrt{r}+\sqrt{q} .
\end{aligned}
$$

Since $r(\tau)=\tau /(\tau+1)$, the function $r$ is continuous and increasing on $[0,1]$ and $0 \leqslant r(\tau) \leqslant 1 / 2$ for all $\tau \in[0,1]$. Thus, assumption (iii) of Theorem 8 is satisfied.

In our case, the constant $K$ is given by

$$
\begin{aligned}
K & =\sup \left\{\int_{0}^{1}|k(t, \tau)| d \tau: t \in[0,1]\right\} \\
& =\sup \left\{\int_{0}^{1} \sqrt[3]{m t^{2}+\tau} d \tau: t \in[0,1]\right\} \\
& =\sup \left\{\frac{3}{4}\left[\sqrt[3]{\left(m t^{2}+1\right)^{4}}-\sqrt[3]{\left(m t^{2}\right)^{4}}\right]: t \in[0,1]\right\} \\
& =\frac{3}{4}\left[\sqrt[3]{(m+1)^{4}}-\sqrt[3]{m^{4}}\right] .
\end{aligned}
$$

In our case, the inequality appearing in assumption (iv) of Theorem 8 takes the form

$$
\begin{aligned}
\|p\|_{1 / 2} & \left(2 K+k_{\beta}\right) \\
= & (\sqrt{r}+\sqrt{q})\left(\frac{3}{2}\left[\sqrt[3]{(m+1)^{4}}-\sqrt[3]{m^{4}}\right]+\sqrt[3]{m}\right) \\
< & \frac{1}{4}
\end{aligned}
$$

It is easily seen that the above inequality is satisfied when, for example, $q=r=1 / 216$ and $m=1 / 128$.

Therefore, using Theorem 8 , we infer that (33) for $q=r=$ $1 / 216$ and $m=1 / 128$ has at least one solution in the space $H_{\alpha}[0,1]$ with $0<\alpha<1 / 2$. 


\section{Conflict of Interests}

The authors declare that there is no conflict of interests regarding the publication of this paper.

\section{References}

[1] K. M. Case and P. F. Zweifel, Linear Transport Theory, AddisonWesley, Reading, Mass, USA, 1967.

[2] S. Chandrasekhar, Radiative Transfer, Dover Publications, New York, NY, USA, 1960.

[3] S. Hu, M. Khavanin, and W. Zhuang, "Integral equations arising in the kinetic theory of gases," Applicable Analysis, vol. 34, no. 3-4, pp. 261-266, 1989.

[4] C. T. Kelley, "Approximation of solutions of some quadratic integral equations in transport theory," Journal of Integral Equations, vol. 4, no. 3, pp. 221-237, 1982.

[5] J. Banaś, M. Lecko, and W. G. El-Sayed, "Existence theorems for some quadratic integral equations," Journal of Mathematical Analysis and Applications, vol. 222, no. 1, pp. 276-285, 1998.

[6] J. Banaś, J. Caballero, J. Rocha, and K. Sadarangani, "Monotonic solutions of a class of quadratic integral equations of Volterra type," Computers \& Mathematics with Applications, vol. 49, no. 5-6, pp. 943-952, 2005.

[7] J. Caballero, J. Rocha, and K. Sadarangani, "On monotonic solutions of an integral equation of Volterra type," Journal of Computational and Applied Mathematics, vol. 174, no. 1, pp. 119133, 2005.

[8] M. A. Darwish, "On solvability of some quadratic functionalintegral equation in Banach algebra," Communications in Applied Analysis, vol. 11, no. 3-4, pp. 441-450, 2007.

[9] M. A. Darwish and S. K. Ntouyas, "On a quadratic fractional Hammerstein-Volterra integral equation with linear modification of the argument," Nonlinear Analysis. Theory, Methods \& Applications, vol. 74, no. 11, pp. 3510-3517, 2011.

[10] M. A. Darwish, "On quadratic integral equation of fractional orders," Journal of Mathematical Analysis and Applications, vol. 311, no. 1, pp. 112-119, 2005.

[11] R. P. Agarwal, J. Banaś, K. Banaś, and D. O’Regan, “Solvability of a quadratic Hammerstein integral equation in the class of functions having limits at infinity," Journal of Integral Equations and Applications, vol. 23, no. 2, pp. 157-181, 2011.

[12] D. D. Bainov and S. G. Hristova, Differential Equations With Maxima, Taylor and Francis, Chapmon and Hall, 2011.

[13] S. G. Hristova and D. D. Bainov, "Monotone-iterative techniques of V. Lakshmikantham for a boundary value problem for systems of impulsive differential equations with 'supremum,"' Journal of Mathematical Analysis and Applications, vol. 172, no. 2, pp. 339-352, 1993.

[14] J. Henderson and S. G. Hristova, "Eventual practical stability and cone valued Lyapunov functions for differential equations with 'Maxima"' Communications in Applied Analysis, vol. 14, no. 3-4, pp. 515-524, 2010.

[15] R. P. Agarwal and S. Hristova, "Strict stability in terms of two measures for impulsive differential equations with 'supremum," Applicable Analysis, vol. 91, no. 7, pp. 1379-1392, 2012.

[16] M. Bohner, S. G. Hristova, and K. Stefanova, "Nonlinear integral inequalities involving maxima of the unknown scalar functions," Mathematical Inequalities \& Applications, vol. 15, no. 4, pp. 811-825, 2012.
[17] A. Golev, S. Hristova, and A. Rahnev, "An algorithm for approximate solving of differential equations with "Maxima'”' Computers \& Mathematics with Applications, vol. 60, no. 10, pp. 27712778, 2010.

[18] J. Caballero, B. López, and K. Sadarangani, "On monotonic solutions of an integral equation of Volterra type with supremum," Journal of Mathematical Analysis and Applications, vol. 305, no. 1, pp. 304-315, 2005.

[19] M. A. Darwish, "On monotonic solutions of a singular quadratic integral equation with supremum," Dynamic Systems and Applications, vol. 17, no. 3-4, pp. 539-550, 2008.

[20] M. A. Darwish and K. Sadarangani, "Nondecreasing solutions of a quadratic Abel equation with supremum in the kernel," Applied Mathematics and Computation, vol. 219, no. 14, pp. 7830-7836, 2013.

[21] J. Banaś and R. Nalepa, "On the space of functions with growths tempered by a modulus of continuity and its applications," Journal of Function Spaces and Applications, vol. 2013, Article ID 820437, 13 pages, 2013. 


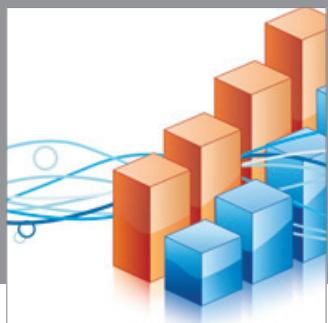

Advances in

Operations Research

mansans

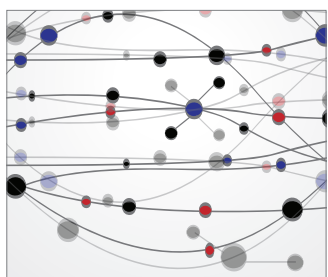

The Scientific World Journal
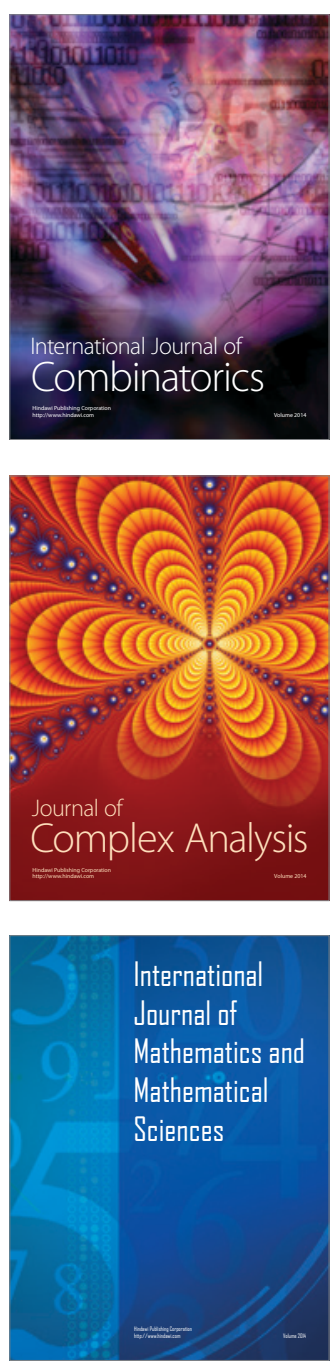
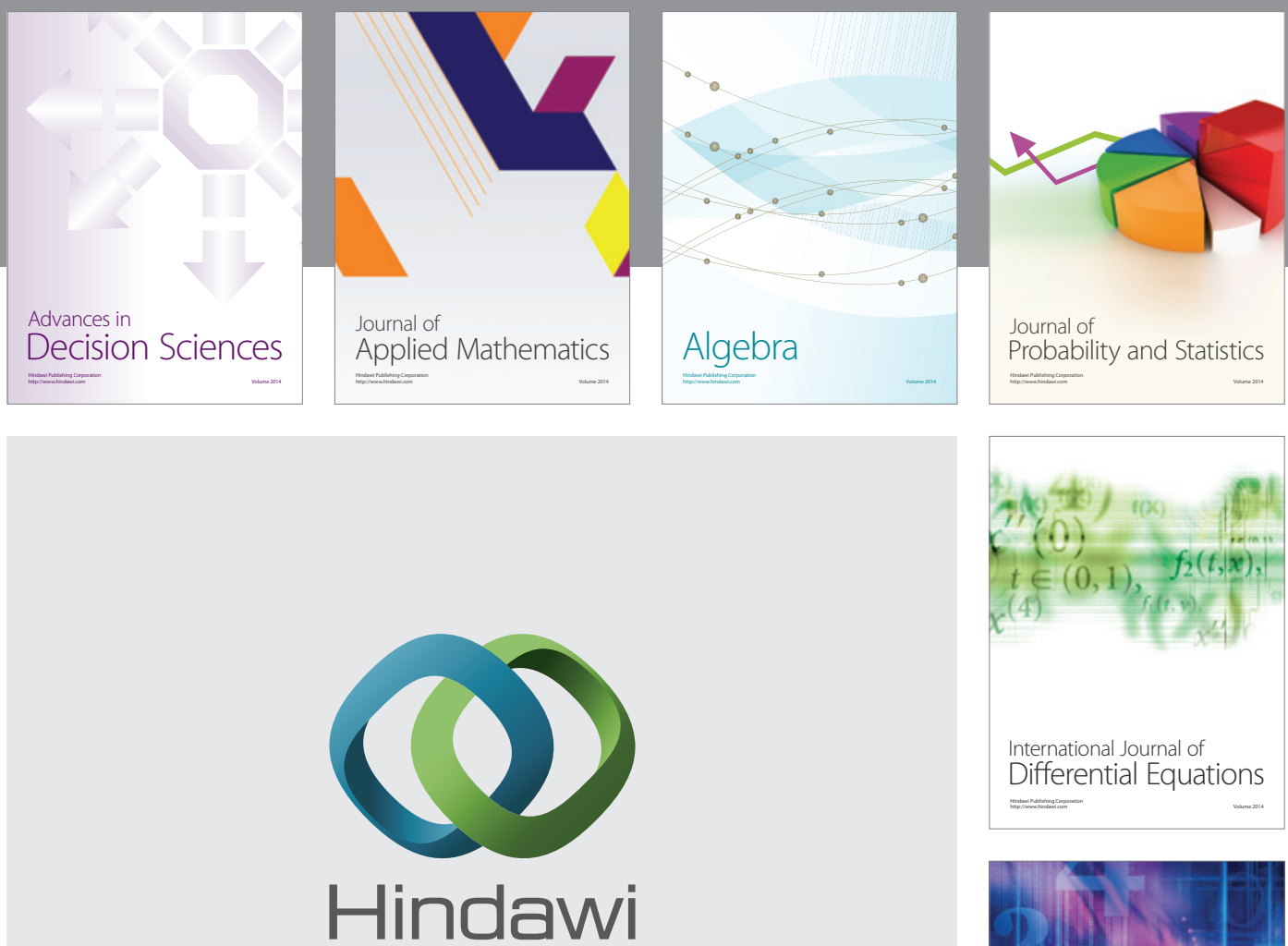

Submit your manuscripts at http://www.hindawi.com
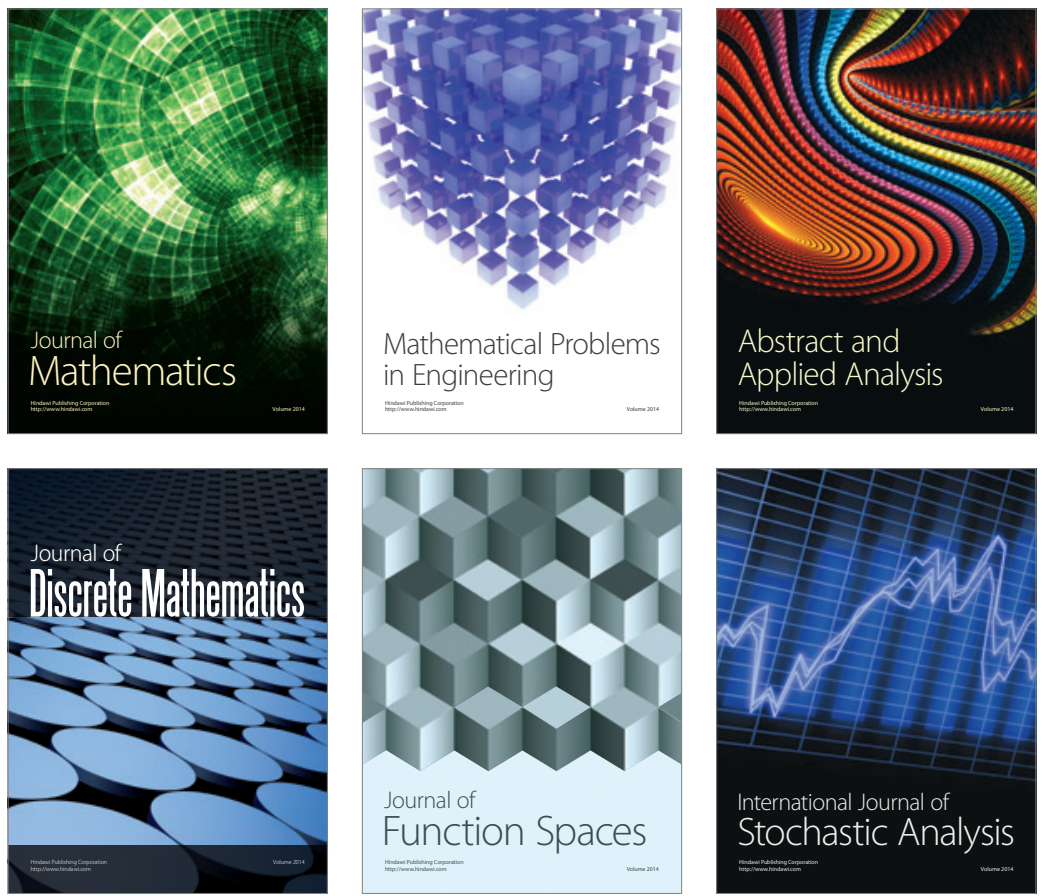

Journal of

Function Spaces

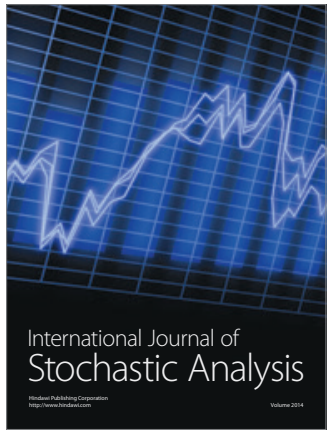

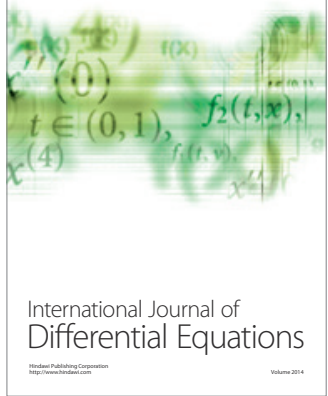
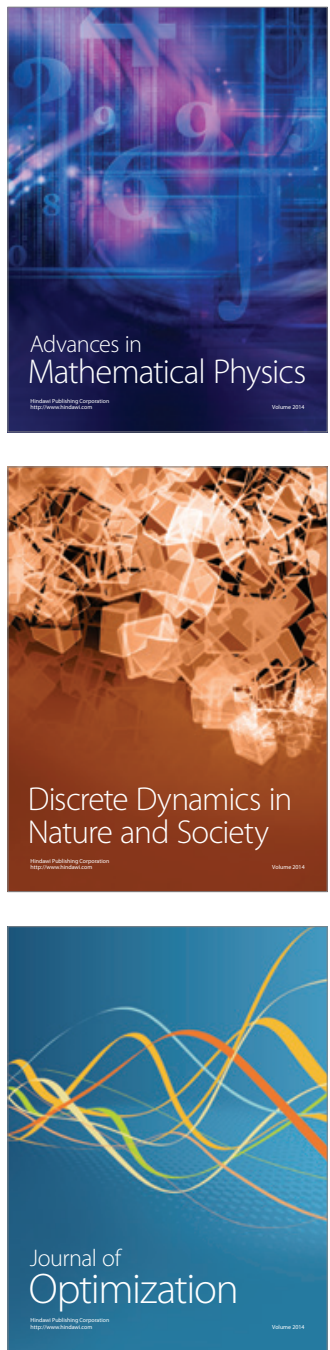